\title{
EXPERT Cancer and the microbiome: REVIEWS \\ potential applications as new tumor biomarker
}

Expert Rev. Anticancer Ther. Early online, 1-14 (2014)

\section{Khan Shahanavaj ${ }^{1,2}$, Ignacio Gil-Bazo $0^{3 \ddagger}$ Marta Castiglia ${ }^{4,5}$, Giuseppe Bronte ${ }^{5}$, Francesco Passiglia ${ }^{5,7}$, Anna P Carreca ${ }^{4,5}$, José Luis del Pozo ${ }^{3}$, Antonio Russo ${ }^{5}$, Marc Peeters ${ }^{6}$, Christian Rolfo*7\$ \\ ${ }^{1}$ Department of Bioscience, Shri Ram Group of College (SRGC), Muzaffarnagar, UP, India ${ }^{2}$ Nanomedicine and Biotechnology Research Unit, Department of Pharma- ceutics, College of Pharmacy, King Saud University, PO Box 2457, Riyadh 11451, Saudi Arabia \\ ${ }^{3}$ Department of Oncology, Clinica Universidad de Navarra, Pamplona, Spain \\ ${ }^{4}$ Molecular Pathology Unit and Phase I- Early Clinical Trials Unit, Oncology Department and Multidisciplinary Oncology Center Antwerp (MOCA) Antwerp University Hospital, Edegem, Belgium \\ ${ }^{5}$ Department of Surgical, Oncological and Oral Sciences, Section of Medical Oncology, University of Palermo, Palermo, Italy \\ ${ }^{6}$ Oncology Department and Multidisci- plinary Oncology Center Antwerp (MOCA) Antwerp University Hospital, Edegem, Belgium \\ ${ }^{7}$ Phase I- Early Clinical Trials Unit, Oncology Department and Multidisci- plinary Oncology Center Antwerp (MOCA) Antwerp University Hospital, Edegem, Belgium \\ *Author for correspondence: \\ Tel.: +32 38213646 \\ christian.rolfo@uza.be}

Authors contributed equally

Microbial communities that colonize in humans are collectively described as microbiome. According to conservative estimates, about $15 \%$ of all types of neoplasms are related to different infective agents. However, current knowledge is not sufficient to explain how the microbiome contributes to the growth and development of cancers. Large and thorough studies involving colonized, diverse and complex microbiome entities are required to identify microbiome as a potential cancer marker and to understand how the immune system is involved in response to pathogens. This article reviews the existing evidence supporting the enigmatic association of transformed microbiome with the development of cancer through the immunological modification. Ascertaining the connection between microbiome and immunological responses with risk of cancer may direct to explaining significant advances in the etiology of cancer, potentially disclosing a novel paradigm of research for the management and prevention of cancer.

\section{KEYWORDS: cancer biomarker • cancer diagnosis $\bullet$ cancer prevention • immunological modification $\bullet$ transformed} microbiome

Cancer, which is known as the proliferation of the host cells in an uncontrolled manner, is a major cause of death worldwide. However, current scientific knowledge is not enough to explain why only a small percentage of people exposed to environment-related carcinogens or bearing specific oncogenic mutations predisposing to cancer develop this disease. Obviously, a combination of certain factors as opposed to a single factor would help in understanding the issue. Among these factors, human microbiome may have an important role. The commensal, symbiotic and pathogenic microorganisms that live within the human body are estimated to outnumber human somatic and germ cells by a factor of 10. Collectively, the genomes of these microbial communities are termed as the microbiome [1]. The human microbiome contains innumerable microorganisms, including bacteria, fungi, protozoa and viruses living in our body $[2,3]$. A very complex and dynamic association between the host and the microbiota arises soon after birth. The microbiome appears to evolve over an individual's lifespan. However, the exact magnitude of its changes is unknown. Perhaps, it is a trend that human microbiome encloses at least 10-times more microorganisms than human cells, accounting for 100-times more genes (microbiome) than the human genome [3-6]. However, this microbiota mostly remains unstudied, leaving almost completely unidentified their effect on human physiology, nutrition, immunity and development. It is noteworthy that microorganisms associate with humans by a non-random process and adapt to specific body habitats [7]. Interestingly enough, the human microbiome from the same site of the body shares more similarities among different individuals than the microbiome from different sites on the same individual. This site-specific colonization is a long process, which has evolved along with the evolution of human beings by various essential physiological and immunological body functions. Overall, the microbiome at a specific site varies with the host genotype, pathobiology (infection or disease status), physiological status, the presence of transient microorganisms, lifestyle and environment, including living state and diet [8,9]. Moreover, one can hypothesize that few species of the 
human microbiome may form idiosyncratic microbial signatures'. These specific signatures may be peculiar to certain body sites and depend on body condition and vice versa [10-14]. Consequently, the normal human microbiome would prevent various diseases, whereas alterations in the normal microbiome creating a transformed microbiome may predispose the individual to numerous conditions.

As previously observed, the commensal microbiota that reside on the host body surfaces and the gastrointestinal tract play an important role in many crucial functions of the host, such as immune response, cancer development prevention and energy metabolism [15,16]. In a previous report, it has been shown that Lactobacillus salivarius, which is an ingredient of human gut microbiota secretes bacteriocin that can protect the mice against invasive foodborne infection of Listeria monocytogenes. This toxin produced by Lactobacillus prevents Listeria infection in mice [17]. Moreover, a recent study has proposed that colonic microbiota may change the host susceptibility to Citrobacter rodentium-induced colitis by modulating inflammation, redox status and ion transporter gene expression [18]. In addition to the aforementioned examples of the protective role of microbiota, some changes in the gut microbiota may allow for the development of several gastrointestinal tract abnormalities [19]. It is reasonably clear that these microbial signatures may be altered under the pressure of determined changes in the body environment.

Impeded regulation in the association between the microbiota and the host immune system may result in a cause of inflammation and development or progression of cancer. The significance of inhabited microbiota in the carcinogenesis of intestinal neoplasia and inflammatory bowel diseases has been evidently showed in animal models recently, where experimental hosts grow colitis in traditional conditions; however, this is not the case when these microbiota are absent in germ-free environments $[16,20,21]$. The cancer-causing agent azoxymethane can trigger the development of colon tumors in colitic IL-10-1acking mice monocolonized with particular bacteria, but stimulating the growth of tumors in germ-free IL-10-lacking mice was unsuccessful [16]. In this review, we will focus on the recent progress in the studies on the enigmatic relationship of transformed microbiome with the development of cancer and its potential application in cancer diagnosis. Furthermore, we will discuss the potential role of the immunological modification and Toll-like receptor (TLR) in the development of cancer and its hypothetical use in cancer therapy.

\section{Evidences for the involvement of the microbiome in the progress \& development of cancer}

Cancer development is a long process that is related to several alterations in the body environment, including physiological, biochemical, immunological and anatomical changes. It could be argued that alterations during the development of cancer due to several modulations in body environment, including immunological, biochemical as well as anatomical alterations, would also manifest an effect on the normal microbiome.
Many studies have confirmed that small alterations in the microbiome from medical origin (antibiotics, vaccination, hygiene etc) or due to host genetics (mutation in IL23R, ATG16L1, IGRM etc), early colonization during delivery and lifestyle may involve etiological or adjuvant roles in the development and progression of cancer (Figure 1A) [22]. Therefore, an altered microbiome may also be used as a strong marker for the detection of various neoplasms, such as pancreatic cancer, colorectal cancer (CRC), cervical cancers, oral squamous cell carcinoma, esophageal cancer, gastric cancer and gallbladder cancer. Recently, a number of studies have identified numerous changes in normal microflora associated with cancer development, and some of these are illustrated in Tabie 1.

Pancreatic cancer is the most common reason of cancerrelated mortality and the fourth leading cause of death due to cancer. Lack of specific detection techniques for pancreatic cancer consistently results in a typical clinical presentation of incurable disease at early diagnosis. Saliva may resolve the issue as an accessible, non-invasive, reliable and efficient diagnostic source. Early stage detection of pancreatic cancer could increase the rate of survival; although, currently no specific biomarker has been shown to be sensitive, reliable and specific for the diagnosis of pancreatic cancer. In a recent study, it was found that Porphyromonas gingivalis, a periodontal oral bacterium, is frequently increased in human pancreatic cancer. This study suggests that patients who have increased levels of antibodies against $P$. gingivalis ATTC 53978, an oral bacterium linked with periodontal tissue destruction, are at a higher risk of pancreatic cancer, whereas a separate group of patients with elevated antibodies against oral bacteria were linked with a lower risk of pancreatic cancer [23]. A report of salivary microbial profiling in pancreatic cancer patients and healthy controls also showed a significant alteration in the salivary microflora. In that study, Farrell et al. identified that the levels of Streptococcus mitis and Neisseria elongata were significantly reduced, whereas the levels of Granulicatella adiacens was increased in cancer patients compared to healthy controls. These data confirm a connection between $G$. adiacens and $N$. elongata and periodontal disease, which has been linked to an increased risk of pancreatic cancer [24]. Farrell et al. also assessed the specificity of the microbial biomarkers using Human Oral Microbe Identification Microarray in another microbial study that had been carried out on lung cancer patients. They found that none of the microbial biomarkers validated in their study showed considerably changed microflora profile in lung cancer [24]. These results confirm that the bacterial biomarkers of saliva are specific for pancreatic cancer and show a potential role for salivary microflora as a non-invasive indicator for the detection of pancreatic cancer. Finally, the results confirmed that the levels of $N$. elongate and S. mitis in saliva are considerably increased in pancreatic cancer patients [24]. Similarly, the tumor most directly associated with oral microbiota is the oral squamous cell carcinoma (OSCC), one of the 10 most prevalent cancers worldwide with an approximate range of $90 \%$ mouth neoplasms originating from oral mucosa. The pathogenesis of 


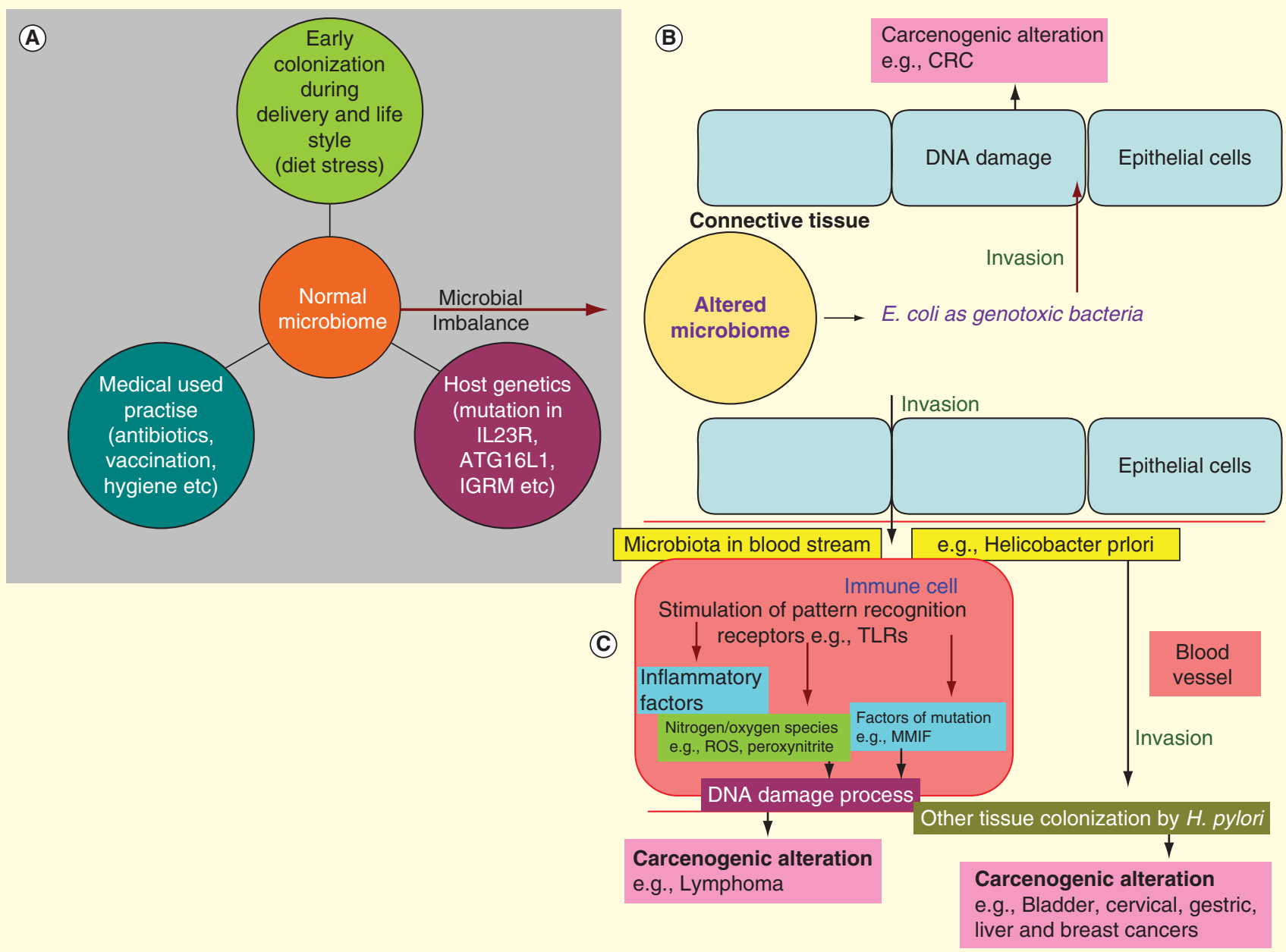

Figure 1. Normal to altered microbiome and carcinogenesis. (A) Many commensal microorganisms permanently reside in the Gl tract and have crucial role in immunity, metabolism, digestion and cancer cure [16,50,51], but it may support cancerous transformation in many cell types through the changes of their microenvironment. (B) E. coli, a genotoxic bacteria, invaded epithelial cells and started their alteration through DNA damage and inflammatory factors in colorectal carcinoma. (C) Genotoxic bacteria (Helicobacter pylori) entered the bloodstream and transformed lymphocytes by DNA damage. The elevated level of pro-inflammatory factors, macrophage migration inhibitory factor, reactive oxygen species and Toll-like receptors may infect several other cell types, resulting eventually in a cause of breast, gastric, cervical, liver and bladder cancers.

OSCC is linked by various abiotic factors, such as heavy alcohol consumption, smoking history as well as many biotic factors, including diverse infections caused by virus, Candida and chronic bacteria. The OSCC surface has been reported to harbor increased levels of Fusobacterium and Porphyromonas compared to contiguous healthy control mucosa.

In a recent study of oral microbiota in tumor and nontumor tissues of patients with OSCC, it was observed that Peptostreptococcus stomatis, S. salivarius, S. gordonii, G. haemolysans, G. morbillorum, Johnsonella ignava and $S$. parasanguinis $I$ were strongly linked with site of tumor, although prevalence of $G$. adiacens was found in non-tumor site. However, S. intermedius existed in approximately $70 \%$ cases of both non-tumor and tumor sites [25]. In a different study of squamous cell carcinoma, a significant increase in 3 out of the 40 organisms was diagnosed compared to healthy controls. These three dominant species were Capnocytophaga gingivalis, Prevotella melaninogenica and $S$. mitis. The increase in these bacteria was predictive in $80 \%$ of OSCC cases and was suggested as a cancer marker [26].

CRC is the second most frequent carcinoma in women and the third most frequent carcinoma in men, with the majority of cases observed in the developed countries. Various factors, such as genetics of the host, diet, environment and alteration in intestinal microbiome have been suggested as a cause for the initiation and progression of CRC in humans. Bacterialmediated infections such as Escherichia coli also play a potentially important role in the development of CRC (Figure 1B) $[16,27,28]$. In a recent study, a significant increase of Desulfovibrio, Erysipelotrichaceae and Fusobacterium was also observed in the intestinal microbiota of CRC [29,30]. Furthermore, in a recent study, it was observed that Roseburia, an anaerobic fecal bacteria, is significantly increased $(\mathrm{p}=0.017)$ in CRC patients of 


\section{Table 1. Alteration in microbiota connected with various type of cancer.}

\begin{tabular}{|c|c|c|}
\hline $\begin{array}{l}\text { Sr. } \\
\text { no. }\end{array}$ & $\begin{array}{l}\text { Type of } \\
\text { cancer }\end{array}$ & $\begin{array}{l}\text { Patients involved in particular cance } \\
\text { related studies }\end{array}$ \\
\hline 1 & $\begin{array}{l}\text { Cervical } \\
\text { cancers }\end{array}$ & $\begin{array}{l}\text { Study involved the vaginal samples of } 68 \\
\text { infected and uninfected woman twins usir } \\
\text { pyrosequencing analysis }\end{array}$ \\
\hline 2 & $\begin{array}{l}\text { Colorectal } \\
\text { cancer }\end{array}$ & $\begin{array}{l}\text { Study involved } 46 \text { patients with CRC and } \\
46 \text { healthy controls from fecal samples }\end{array}$ \\
\hline
\end{tabular}
cancer $\quad 46$ healthy controls from fecal samples

Study was performed on 29 patients with colon adenomas, 31 with colorectal cancer, 34 symptomatic but normal colonoscopy results and 31 asymptomatic patients as controls

Experiment involved biopsy samples of 21 patients with adenomas and 23 without adenomas as controls

Study was performed on feces specimens of 60 CRC patients and 119 healthy controls

3 Oral cancer

Case study was carried out using 229 oral squamous cell carcinoma (OSCC) free and 45 OSCC patients and their consequent evaluation for 40 oral bacteria by DNA-DNA hybridization

Tissue samples were used from 46 oral cancer and three precancerous leukoplakia

$4 \quad$ Esophageal cancer

$5 \quad$ Gastric cancer

Gall bladder cancer
Biopsy and aspirate from 7 patients with Barrett's esophagus (BE) and 7 control with $\mathrm{BE}$, and their subsequent analysis by culture followed by $16 \mathrm{~s}$ rRNA gene sequencing involved in this study

DNA samples were used from 15 esophageal cancers, 16 lung cancers, 43 gastric cancers, 10 cervical cancers, 10 colorectal cancers, 14 renal cell carcinomas and 19 bladder cancers

Tissues of esophageal carcinoma, the corresponding normal tissues and saliva were collected from patients in China, Japan, Italy and France followed by analysis of bacterial diversity by 16 s rRNA gene

Study involved 260 controls, 227 esophageal adenocarcinoma, 224 Barrett's esophagus and 230 reflux esophagitis patients

Biopsy samples of 2019 gastric cancer patients were collected from Brazil

Tissue and blood samples of 54 gallbladder cancer patients were analyzed though nested PCR

Study was carried out using bile culture in 390 patients, including 65 with gallbladder carcinoma, 125 cholelithiasis along with 200 control samples
Alterations in the microbiota

Increased levels of Fusobacteria, such as

$[132,135]$

Sneathia spp and decreased levels of Lactobacillus spp. were found in HPV-infected cervical cancers patients

Increased proportions of Enterococcus, Escherichia/Shigella, Bacteroides fragilis, and Klebsiella, and decreased level of Roseburia and members of family Lachnospiraceae were observed in CRC

Presence of intracellular $E$. coli increased in patients with adenoma and CRC

[134,137]

Increased levels of Proteobacteria, Faecalibacterium and Dorea, whereas decreased levels of Coprococcus and Bacteroides were observed in adenomas patients

The level of Bacteroides/Prevotella was increased in $C R C$ patients as verified by quantitative $P C R$ Increased levels of Streptococcus mitis, Capnocytophaga ochracea, Eubacterium saburreum and Leptotrichia buccalis were found in OSCC

Higher levels of Streptococcus anginosus was observed in OSCC

High levels of Campylobacter concisus and C. rectus were observed in Barrett's esophagus

Increased level of S. anginosus was found in only esophageal cancer and gastric cancer tissues

Increased proportion of Treponema denticola, S. anginosus and S. mitis were found in esophageal cancer

Decreased level of $H$. pylori was observed in esophageal cancer

Increased proportion of Helicobacter pylori was observed in gastric cancer

Elevated level of $H$. pylori was observed in 33\% of gallbladder cancer patients

Increased level of Salmonella typhi and

S. Paratyphi was observed in bladder cancer 
Table 1. Alteration in microbiota connected with various type of cancer (cont.).

\begin{tabular}{llll}
$\begin{array}{l}\text { Sr. } \\
\text { no. }\end{array}$ cancer & $\begin{array}{l}\text { Patients involved in particular cancer- } \\
\text { related studies }\end{array}$ & Alterations in the microbiota \\
\hline 7 & $\begin{array}{l}\text { Pancreatic } \\
\text { cancer }\end{array}$ & $\begin{array}{l}\text { Chronic pancreatitis samples of } 27 \text { patients and } \\
28 \text { resectable pancreatic cancer with } 10 \text { healthy } \\
\text { controls were validated for bacterial candidates } \\
\text { through qPCR }\end{array}$ & $\begin{array}{l}\text { High levels of Neisseria elongata and S. mitis } \\
\text { were observed in pancreatic cancer }\end{array}$
\end{tabular}

China [31]. In another report, it was observed that Fusobacterium nucleatum, an exceptional member of fecal microbiota, is frequently over-represented among patients with CRC [32,33].

The enigmatic connection between gut microbiota and cancer has opened a new door of research regarding cancer detection, prevention and may further lead to the development of innovative treatments. The important role of Helicobacter pylori in the progression of gastric cancer, bladder cancer, OSCC, cervical cancer, hepatocarcinoma and mucosa-associated lymphoid tissue lymphoma are well established (FIGure 1C) [34-39]. For this reason, the presence of this organism should be considered a relevant risk factor for developing gastric carcinoma. In a recent study of CagA-positive strains of $H$. pylori, a positive association of such strain was observed with esophageal squamous cell carcinoma in non-Asian population and an inverse association in Asian population [40]. Moreover, a study in esophageal cancer showed that this neoplasia may be linked to a frequent infection of Treponema denticola, S. mitis and S. anginosus. Furthermore, self-regulating culture methods used on the saliva of esophageal cancer patients and healthy individuals revealed a strong association between group G streptococcal infection and esophageal cancer [41]. In addition, similar results have been also observed in CRC with regards to its potential association with group G streptococcal infection [42]. Patients with Barrett's esophagus are also at a higher risk of developing esophageal adenocarcinoma but not squamous cell cancer. These patients show elevated levels of Campylobacter species (C. rectus and C. concisus) in esophageal biopsies and aspirate samples. These microbial markers may also pave the way for diagnosing Barrett's esophagus [43]. The examples reported here suggest a clear association of microflora changes and cancer development. Although there is considerable research in this area, it is still insufficient and more efforts are required to fully understand the changes in microbiota during cancer development and progression.

Several studies demonstrate that diverse alterations in the microbiome can increase or decrease cancer susceptibility and development by different mechanisms, such as influencing the genomic stability of host cells, lateral gene transfer, alteration in growth hormones, biochemical and enzymatic alteration, cell surface receptor modification, modulating inflammation and making various metabolites that act as histone deacetylase inhibitors to epigenetically control gene expression of host. Although there are several causes behind the alteration of microbiome and the development of cancer, as discussed above, we shall now explore the immunological modification and its potential role in the transformation of the normal microbiome into a cancerous microbiome. Various cancers arise from inflammation and chronic irritation in sites of infection.

\section{Immunological modifications from a normal microbiome to a cancerous microbiome}

The mechanisms of development and progression of cancer are very complex through diverse microbial communities. Virchow described the connection between inflammation and the possibility of developing cancer in 1863 [44] based on the fact that carcinogenesis tends to arise at the site of chronic inflammation. Many studies suggest that this mechanism involves a relation between chronic inflammation, direct effects of microbial communities on host cell physiology and, eventually, alterations in the tissue equilibrium. Alterations in the microbiome may affect both systemic and local inflammation. Inflammation is in close relation to progression, development or even cure of cancer, although it remains uncertain whether commensal microbiota influence inflammation in the microenvironment. In a recent report, it was observed that tumor-infiltrating myeloid-derived cells reacted poorly during cancer therapy of germfree or antibiotics-treated mice $[45,46]$. In fact, these reports showed that the existence of two commensal microbiota, Alistipes and Ruminococcus, positively correlated with tumorassociated myeloid cells secreting TNF- $\alpha$ or IL-17, thus increasing anticancer effect. These findings assert the fact that 'immunostimulatory microbiota' could be utilized to improve the adverse effect of microbiota reduction in patients or even optimize the response of anticancer drugs [45,46]. Various epidemiologic reports have strongly showed that also chronic inflammation is linked to an enhanced risk of cancer [47,48]. It is assessed that approximately 2 million cases of cancer are caused by infectious agents annually [49]. Both mutualistic and nonmutualistic microorganisms residing permanently in the gastrointestinal tract, which play an important role in immunity, digestion and prevention of cancer [50,51], may be activated provoking inflammatory responses in different cancer types, such as lymphoma and leukemia [52-54].

The inflammatory responses that effectively reduce pathogen-associated molecular patterns (PAMPs) and danger-I damage-associated molecular patterns (DAMPs) are dynamically stopped and the process of healing initiated thereafter. Previous studies showed that the phagocytosis of apoptotic cells increases the level of anti-inflammatory mediators supporting 
the anti-inflammatory response [55]. However, current findings demonstrate that impaired phagocytosis supports inflammation and employs other immune cells [56], proposing a crosstalk between the immune cells and the inflamed tissue. That crosstalk would be arbitrated by the pro- and anti-inflammatory factors abundantly generated by both infiltrating and inhabitant cells $[57,58]$. Experiments performed in a rat carrageenan-sponge implant model have showed a change from tissue repair connecting tissues. Pro-inflammatory and anti-inflammatory effects may depend on the site of inflammation in such microenvironment $[59,60]$. These factors have been detected in humans also and comprise, for example, alterations in growth factor- $\alpha$ [61], metalloproteinases [62], prostaglandin E2 [63], nitrogen species and reactive oxygen [64,65].

Inflammatory responses are stopped following the removal of pathogens; however, they can persist in the case of chronic infection, resulting in tissue fibrosis and carcinogenesis by the establishment of adverse inflammatory cycles [66-68]. The characteristics of chronic inflammation are inflammatory foci directed by plasma cells, lymphocytes and macrophages, generating a high level of cytokines, growth factors, chemokines, nitrogen species and reactive oxygen, which may be responsible for incessant tissue damage [69,70]. Interestingly enough, high levels of nitrogen species and reactive oxygen liberated in such pathological states may generate mutagenic agents, such as peroxynitrite $\left(\mathrm{ONOO}^{-}\right)$, for example, which interacts with DNA and develops mutations and injures in proliferating cells [71,72]. These DNA alterations may cause a predisposition to neoplasia [73]. In addition, the elevated levels of growth factors and pro-inflammatory cytokines, including TNF- $\alpha$ and macrophage migration inhibitory factor, produced by T-lymphocytes and macrophages may aggravate DNA damage [74]. Macrophage migration inhibitory factor interferes with the crucial cell cycle signaling pathway cyclin/Rb/E2F and plays a key role in tumorigenesis [75], also impairing p53-dependent protective responses and thus giving rise to oncogenic mutations [76]. In addition, the microenvironment of inflammation is appropriate for the proliferation and survival of neoplastic cells [57,58], demonstrating that the modulation of factors increasing chronic inflammation may have anticancer effects. An enigmatic study has showed that eradication of $C$. jejuni, a Gram-negative bacterium, suppresses the inflammation-mediated lymphomagenesis in immuno-proliferative small bowel disease at its initial stage [52]. Similarly, another report has showed that several commonly used antineoplastic agents that cause DNA damageinduced apoptosis in highly sensitive cells, such as altering cells, have antimicrobial activities against Acinetobacter spp. [77]. Acinetobacter spp. are non-microbiota pathogens connected with various solid tumors [78-80] and blood cancers [77]. Such agents comprise the alkylating-like agent cisplatin, the alkaloid vincristine and the anthracycline doxorubicin. Recent results show that Acinetobacter spp. become as sensible to DNA damage as altering cells during carcinogenesis due to inflammation, showing the complexity of the interactions between altering cells and cancer-related pathogens. TLRs are transmembrane proteins able to recognize a constituent pathogen. They are involved in identifying endogenous danger signals and play an important role in eliciting innate immune response against infecting pathogens. Future studies dealing with the relationship between bacteria and altering cells may give novel insights into bacteria-mediated inflammation and the consequent processes of carcinogenesis.

\section{TLRs, pathogen-triggered inflammation, signaling \& cancer}

Gram-negative bacteria and organisms such as bacteroidetes, proteobacteria, fusobacteria and spirochaetes produce lipopolysaccharide (LPS) a specific immune-activating ingredient, which can stimulate innate immune responses that may direct various diseases, including cancer [81]. LPS connects to TLR4 of innate immune system, leading to the activation of $\mathrm{NF}-\kappa \mathrm{B}$. As a result, the proportion of activated NF- $\kappa \mathrm{B}$ is enhanced in patients with esophageal adenocarcinoma and reflux esophagitis and Barrett's esophagus. Enhanced levels of activated NF- $\kappa \mathrm{B}$ are linked with increased amounts of TNF- $\alpha$ and inflammatory cytokines, including IL-1b, IL-6 and IL-8 [81]. The activation of nitric oxide synthase reduces the basal tone of the lower esophageal sphincter, which, in excess of extended periods of time, raises the threat for reflux and its consequences [82]. Furthermore, LPS has been demonstrated in rodents to delay gastric emptying, which enhances the level of gastric content refluxed into the esophagus [83]. Thus, the microbiome of the esophagus might be managed with probiotics, antibiotics or NF- $\mathrm{KB}$-specific host cell pathway inhibitors to prevent cancer or other disorders in this anatomical location. TLRs play a crucial role in the early innate immune response to invading pathogens and are involved in observing endogenous danger signals through the sensing of PAMPs, mainly via myeloid differentiation primary response gene $88(M y D 88)$ adaptor protein [84,85]. In general, most bacteria can provide a source of ligands for TLRs that may act as target cell membrane receptors [86]. The signaling of TLR/MyD88 checks the dissemination of bystander bacteria to insider tissues during infection with Clostridium difficile. This effect is exerted by activating neutrophil and monocyte employment to the lamina propria of the intestine by mechanisms involving CCL2/CCR2 (C-C receptor type 2) and CXCL1 (C-X-C motif ligand 1)/ CXCR2 (C-X-C motif receptor 2) signaling pathways. In fact, the death rate is markedly enhanced in MyD88-lacking mice following the infection of $C$. difficile [85]. Genetic silencing of pattern recognition receptors signaling defends against chronic inflammatory-mediated diseases [87], demonstrating that perturbations of microbiota-pattern recognition receptors interfaces may promote inflammation.

Bacterial sepsis may be a cause of translocation of the microbiota from the intestine into the systemic milieu [88,89]. Short leucine-dominant proteoglycans, including decorin and biglycan, may coordinate TLRs crosstalk during the course of inflammation [90,91]. Septic inflammation induces initial 
responses that comprise the activation of the decorin gene; the level of decorin protein is elevated in mouse models as well as plasma of septic patients, and high levels of decorin enhance the expression of pro-inflammatory molecules [90]. An increased expression of pro-inflammatory molecules has been connected with tumor progression and poorer clinical outcomes in the case of hepatocellular carcinoma [92].

Data in literature suggests that TLRs are crucial players in pathogen-mediated inflammation-induced carcinogenesis. Uronis et al. [93] have showed that bacteria-triggered inflammation could have a role in the evolution of adenoma to invasive carcinoma in IL-10 exposed azoxymethane ( $A O M$ oncogene) knockout mice, an example of colitis-linked CRC. This study has showed that $62 \%$ of $A O M-I L-10(-/-)$ mice with colon tumors versus just $20 \%$ in AOM-wild-type mice develop multiplicity of tumors directly linked with the existence of colitis. Surprisingly, IL-10 (-/-) mice mono-linked with the bacterium Bacteroides vulgatus demonstrated a considerably decreased multiplicity of colitis and colorectal tumors, whereas germ-free AOM-treated IL-10 (-I-) mice showed no tumors. Furthermore, $M y D 88(-/-)$ or AOM-treated IL-10 (-/-) mice showed a decreased TNF- $\alpha$ and IL-12p40 mRNA expression, an identified factor of IL-12 and IL-23. These mice displayed no signs of tumor growth, showing that the pathway of TLR/ MyD88 is necessary for microbiota-mediated growth and progression of colitis-linked CRC. Some evidences support that inflammation resulting from the immunologic response to chronic bacteria and bacterial toxin exposure may play a key role in gastrointestinal and oral carcinogenesis [94-96]. The essential gut microbiota, B. fragilis, utilizes TLRs pathways to establish host microbial symbiosis. This microorganism produces polysaccharide A (a symbiosis factor), which induces several signaling pathways to support immunologic tolerance by TLR2 receptors on $\mathrm{CD}^{+} \mathrm{T}$ cells [97]. The overexpression of TLR molecules is a significant phenotype present in cancer development. Interestingly enough, increased overexpression of TLR3, TLR4, TLR7 and TLR9 molecules has been identified in esophageal squamous cell carcinoma cells compared with normal counterparts [98,99]. Overexpression of TLR4 and TLR9 is similarly observed in lung cancer samples with a significantly positive correlation between TLR4 expression levels and lung cancer [98]. These findings indicate that TLRs play a dual role in the host's defense against infection and in tissue homeostasis maintenance by directing inflammatory and tissue repair response to injury [100]. The inflammatory damage repair pathway may ultimately lead to carcinogenesis through chronic inflammation due to an altered microbiota [101]. The effect of microbiota constituents on chronic intestinal inflammation and colon cancer formation has been shown in gnotobiotic animal models [102]. In conclusion, TLRs overexpression may require more symbiotic factors to initiate immunologic tolerance, severely influencing normal microbiota as a result of the inflammatory response at the tumor site.

Normal microflora may also affect infection of potential pathogens by a specific mechanism called colonization resistance [103]. In contrast, certain organisms of the microbiome may contribute to reduce the incidence of cancer. A probiotic bacterium, Bacillus polyfermenticus, has been shown to impair the growth of human colon cancer cells by the inhibition of ErbB2 and ErbB3 receptors [104]. The specific mechanisms behind the association between bacteria and cancer development include immune evasion, chronic inflammation and immune suppression [36,105]. Although it is not easy to cover the entire immunological effects leading to the change of normal microbiota, it can be supposed by these interactions that the impairment of normal microbiota is strictly related to carcinogenesis.

TLRs identify PAMPs of bacteria, viruses and parasites in the endolysosomes of TLR3, 7-9 and 10 and in extracellular environment of TLR1, 2, 4-6 and 11. TLRs can attach many PAMPs/DAMPs and it is thought that they may even attach regular 'self-' molecules including fibrinogen and heat shock proteins, suggesting a relation between the stereotypic inflammatory response mediated through TLRs and autoimmune diseases [106,107]. The signaling of TLRs is transduced in the cytoplasm through the Toll/IL-1 receptor domain (TIR), which is the site for docking to the cytoplasmic adaptor proteins of TIR that are important in coordinating the pathways of signal transduction after TLR as well as IL-1 receptor activation. The molecules of TIR adaptor mainly contain signaling proteins such as TRIF, TRIF-related adaptor molecule, MyD88, MyD88 adaptor-like as well as negative regulator of TLR pathways sterile-alpha and Armadillo motif containing protein $[108,109]$. All TLRs connect to the adaptor MyD88 except TLR3, but either as IL-1R (TLR5, 7-11, and heterodimeric TLR1-TLR2 and TLR2-TLR6) directly or in connection with MyD88 adaptor-like/TIR domain-containing adaptor protein (TIRAP).

TLRs signaling role in pathogen-mediated inflammation and progression of cancer may be a very complex process. For example, Smolinska et al. [84] have examined the possible role of Src family kinases on TLRs signaling through the key human macrophages along with adenoviral overexpression. The report showed that the Hck a member of Src kinase has a crucial role in LPS/TLR4-induced production of TNF- $\alpha$ and IL-6. Furthermore, studies have suggested that Hck may mediate TLR4-induced transcription of TNF- $\alpha$ and IL- 6 by TLR4 activation through a specific mechanism that does not involve MAPK and NF- $\mathrm{KB}$ pathways. However, it could also direct to p38 MAPK-dependent activator protein (AP-1) connecting with a complex of JunD, and c-fos. Recent reports involving subcutaneous or intraperitoneal injection of the TLR3 interactor polyinosinic-polycytidylic acid (polyI:C) to lung carcinoma implants in mice have revealed that tumor regression is induced through TLR3 signaling by transforming tumor-assisting macrophages into tumor suppressors [99]. Thus, the better understanding and characterization of the TLRs-mediated signaling pathways may offer new therapeutic targets against pathogen-triggered cancers. 


\section{Targeting TLR: is there a therapeutic chance?}

As previously described, there is an emerging evidence for the role of microbiota and TLRs in cancer. TLRs pathway activity leads to the expression of pro-inflammatory cytokines, Type I IFN-1 and chemokines [110]. Under normal circumstances, only a subset of TLRs is detectably expressed in the intestinal epithelium. The deregulated immune response to gut microbiota is thought to play a relevant role in CRC carcinogenesis. This response is mediated also by TLRs; the deregulated signaling of TLR pathways can ensure a pathogenic immune response to normal microbiota [111]. Thus, the contribution of TLRs to tumorigenesis and therapeutic interventions in CRC is under rigorous investigation.

Recently, several Phase I and II clinical trials have been registered to examine the prophylactic and therapeutic potential of TLR agonists/antagonists in various cancers [112]. For example, in melanoma and basal-cell carcinoma patients, intra-tumor injection of PF-3512676 (a TLR agonist) was shown to be able to induce regression of skin lesions [113,114]. PF-3512676 has been also tested in advanced renal cell carcinoma [115] and advanced cutaneous $\mathrm{T}$ cell lymphoma [116]. Imiquimod is a TLR7 agonist that seems to have some activity when it is used topically for the treatment of several premalignant and malignant skin diseases [117], but it is not effective when given by systemic injection, despite some encouraging results obtained in mouse models [118]. These new agents are currently being used in conjunction with other treatments to enhance the anti-tumor immune response. However, due to their potential role in the pathogenesis of the disease, it is plausible that they exert a nonimmune-mediated effect on tumor formation, growth and treatment. The use of these new kind of drugs is still under investigation but there are promising expectations.

\section{Suggestions \& perspectives for the development of microbial signature}

We suggest that the microbiota composition can be helpful for a strong immune response or even protect a person from several diseases, including cancer. Various factors can contribute to the transformation of microbiome, such as host genetics phenotype, medical practices, lifestyle and early colonization during delivery. A person with mutations in genes involved in the regulation of the immune system or pro-inflammation pathways can develop unrestrained inflammation in the host tissue. It is feasible that inflammation alone affects the equilibrium of the microbiota, twisting it in support of pathobionts. On the other hand, a person could 'select' or eliminate the colonization of particular microorganisms. Different pieces of evidence showed that a crucial complex bacterial community lives in the human body, and its changes may play a key role as an indicator for individual cancer susceptibility and development [119]. The complex architecture of this bacterial community generally hinders the development of cancer markers. In addition, the assessment of most micro-organisms is complicated by their lack of suitability for culture [120]. Moreover, inter-individual inconsistency in the microbiome due to host pathobiology, physiology, lifestyle and environment also raises queries of this method in cancer prediction and detection [121]. Conversely, the feces samples of 30 patients demonstrated stable and unique bacterial community profiles [122]. In brief, attempts to identify specific microbial markers from such a large microbiome may contribute to solving this problem. In a recent study, deep ribosomal RNA sequencing of CRC tissue and adjacent non-malignant mucosa revealed prominent differences in microbial colonization patterns between these two sites. CRC tissues demonstrated consistent and increased presence of a subclass of Coriobacteriaceae, especially genera Slackia and Collinsella, although members of Enterobacteriaceae, including Citrobacter, Shigella, Cronobacter, Kluyvera, Serretia and Salmonella sp. were undercharacterized [14]. Because most of the microorganisms in the microbiome cannot be kept in culture, studies to unravel the complex architecture of human microbiome involve culture-independent techniques. However, the isolation of microbial DNA and RNA from normal mucosa and adenomatous polyps also revealed that microflora changes at the mucosal surface in colonic adenomas and this could possibly act as a potential factor facilitating cancer development [123].

Many issues remain to be cleared. First, the existing microbiota of host should be completely diagnosed through metagenomic sequencing approach on whole genes of bacteria to characterize microorganisms promoting healthiness or diseases. Several investigations in this direction are in progress in different regions of the world including the USA and Europe. Potentially transformed microbiota could be identified in patients with several types of cancer and examined in many research models. Whether the change in the existing microbiota directly or indirectly alters the path of disease is a crucial issue and adequate studies are needed to address this question. Other aspect is whether the present information acquiesced by the metagenomic sequencing is enough to begin efficient experiments in which a reason/effect relation could be developed using particular animal models. What would be the productivities of efficient studies using transformed microbiota acquired from germ-free animal models and many disease conditions inflammation and cancer? The characterization of consortium of microbiota linked with specific pathological states illustrates a very important target, this crucial action is not sufficient to entirely understand the potential role of the microbiota in healthiness and disease of individuals. Thus, it is important to investigate the risk and feasibility of alteration of human microbiome and its ability as a novel approach to identify and treat cancer. Although information on this approach is limited, ongoing research in this direction will surely lead to a microbebased novel area of cancer detection.

\section{Limitations \& applications of transformed microbiome in cancer detection}

Finally, it can be concluded that this rapidly growing field of research is bringing critical understanding of the microbiome alteration and its potential role in cancer, disclosing the door 
to new strategies for detection, prevention and treatment of cancer. However, the multi-faceted nature of the microbiome and the reproducibility of microbial signatures under circumstances of individual changeability is a strong constraint before the microbiome modification can be suitably corroborated as an indicator of cancer. Strict detection of stable signatures not influenced by individual pathobiology, physiology, environment and lifestyle are required before the application of these markers on a routine or daily practice basis. Extensive clinical appraisal through retrospective, prospective and randomized clinical trials will generate a cancer indicator possibly more appropriate for early cancer detection. In addition, populationbased assessment is also needed to identify alterations in microbial change in populations with cancer. In view of the discussed drawbacks and lower diagnostic sensitivity compared with routine cancer markers, these indicators should be employed in combination with other known markers, biopsy, imaging, molecular diagnosis and other appropriate clinicopathological information before any conclusions regarding their utility [124]. A relevant limitation is that microbiome is linked to mucosal surfaces of the gut, the urogenital tract and the respiratory airways [125]. So, we could speculate that epithelial cells are highly prone to change during cancer development. In short, we may presume that its application for the cancer derived from epithelial cells is very limited. Sampling bias is another issue that requires consideration before developing a reasonable appraisal of this approach. The microbiome of non-malignant mucosa and adjacent colonized colon tumor tissue from one individual demonstrates a marked difference of the microbiota pattern [14]. Thus, this heterogeneity requires a strong validation of microbiome sampling for cancer detection.

However, nucleic acid, metabolites, proteins and certain processes such as apoptosis and proliferation are well-characterized factors included in the category of cancer detection biomarkers [126]. However, an ultimate, reliable and cost-effective diagnostic marker for cancer detection needs to be in conjunction with a highly specific and significantly sensitive assay [124]. The transformed microbiome signatures may prove to be an outstanding cancer detection indicator, with further use in treatment strategies under various circumstances due to its intricate association with cancer. As we know, protein-based cancer biomarkers usually comprise mass spectroscopy based on peptidomics and proteomics profiling (low-molecular-weight serum/plasma proteins as cancer indicators). However, the effectiveness of plasma or serum-based cancer markers is dependent on its proper sample collection and storage. Otherwise the processing of flawed samples can produce biased results [124]. In fact, the National Academy of Clinical Biochemistry Laboratory Medicine Practice Guidelines do not recommended mass spectroscopy-based serum proteomics profiling for clinical purposes [127]. Serum biomarkers are dependent upon the level of expression of particular marker proteins, post-translational modifications, their stability and association with other proteins [124]. Furthermore, the proteolytic activity of serum also significantly affects low-molecular-weight proteins [128]. Thus, it could be expected that the signatures of normal microbiomes may compensate for the drawbacks of serum protein-based biomarkers. Thus, careful validation of the human microbiome might result in the identification of stable signatures while simultaneously addressing all concerns.

Microarray technology is also an exceptional tool to identify an altered expression of cancer regulatory genes [129]. Despite its high sensitivity, the specificity of this technology as a prognostic marker is unstable, and scientists recommend repeating random sampling validation before the application of these markers in daily clinical practice [130]. Because of the comparable influence of the microbiome in cancer prognosis, its potential as a prognostic factor requires specific evaluation. Thus, it can be expected that more studies regarding these subjects will increase the value on driving forces of cancer and support the expansion of new reliable and sensitive microbiome-associated diagnostic techniques and therapeutic interferences.

\section{Expert commentary}

The majority of the comprehensive studies pertaining to the assessment of cancer etiology and the potential of the human microbiome for the detection and prevention of cancer demonstrate its important role in cancer progression and development. However, the complexity of the human microbiome and allied research progress are ahead of our imagination. Hence how microbiome alteration functions for cancer detection requires rigorous evaluation before establishing its reliability as a cancer biomarker. Various current studies make it clear that alterations in the microbiome are not only linked to an individual's vulnerability to cancer but also its role in cancer diagnosis [131]. Our awareness of the human microbiome is still in its early stages. However, worldwide efforts coupled with technical advancement can open the door to a better understanding of the microbiome composition and the metabolic activities and ultimately toward the development of new microbe-based advanced therapeutics and cancer indicators. One way to achieve this goal would be to routinely search for specific pro-oncogenic pathogens in all patients diagnosed with certain cancer types within a multidisciplinary, multicenter and multinational consortium program. That approach has been already used by the VALGENT study group in the case of the research that correlates the HPV infection and the presence of cervical lesions [132,133]. That enormous effort may definitely contribute to a better knowledge on how different microbiome alterations can facilitate or prevent cancer development. For that purpose, high-throughput technology would be required to be able to molecularly characterize those neoplasms and their respective potentially causal pathogens. Finally, different in vitro and in vivo assays in better models should be developed to ensure the mechanistic validation of the findings derived from that large population-based observation. In addition, all patientderived samples should be thoroughly scrutinized looking for potential microbiome-based biomarkers of cancer presence and progression to standard therapies [134]. The most remarkable challenges we envision in pursuing that goal would be the joint 
efforts between different science fields and experts, the proper development of the technology resources required for this endeavor and the adequate funding to make that program a reality. Conceivably, the time has come to state that alterations in the microbiome signature can change traditional cancer biomarkers but this approach will require sound appraisal to escort it into the next exciting frontier of cancer diagnostics.

\section{Five-year view}

In the past few months, diverse immunological approaches (i.e., PDL-1 targeting) have shown astonishing results in the management of different cancer types, such as melanoma or non-small cell lung cancer. These observations have definitely remarked the critical role of the immune system in the successful treatment of cancer.

After the tremendous development of immunotherapy against cancer, we envision that microbiome-driven clinical trials will be part of the clinical research armamentarium against cancer in the next 5 years. As a crucial part of that clinical research, novel microbiome-derived biomarkers will be under development. In addition and according to the fast evolvement of new sequencing technologies, one can easily conceive that the microbiome molecular profile will be used not only for cancer prevention but also for cancer biological treatment tailoring.

\section{Acknowledgements}

The authors are thankful to C Zaharie, Scientific Expert Status, Health Canada Scientific Experts Database, Ottawa, Canada, for critical review and valuable suggestions for improving the quality of this manuscript.

\section{Financial \& competing interests disclosure}

The authors have no relevant affiliations or financial involvement with any organization or entity with a financial interest in or financial conflict with the subject matter or materials discussed in the manuscript. This includes employment, consultancies, honoraria, stock ownership or options, expert testimony, grants or patents received or pending, or royalties.

No writing assistance was utilized in the production of this manuscript.

\section{Key issues}

- Normal human microbiome may prevent various diseases, including cancer, whereas alterations in the normal microbiome creating a transformed microbiome may predispose the individual to numerous conditions, man of them related to cancer.

- The association between microbiota and the host immune system may result in a cause of inflammation and development or progression of cancer.

- Most of the available evidences are still to be validated and confirmed.

- A routine search for specific pro-oncogenic pathogens in all patients diagnosed with certain cancer types within a multidisciplinary, multicenter and multinational setting would definitely boost this research field.

- Further development of new high-throughput sequencing techniques is warranted to facilitate a molecular characterization of different neoplasms and their respective potentially causal pathogens.

\section{References}

1. Sonnenburg JL, Fischbach MA. Community health care: therapeutic opportunities in the human microbiome. Sci Transl Med 2011;3:78ps12

2. Human Microbiome Project Consortium. Structure, function and diversity of the healthy human microbiome. Nature 2012;486:207-14

3. Reyes A, Haynes M, Hanson N, et al. Viruses in the faecal microbiota of monozygotic twins and their mothers. Nature 2010;466:334-8

4. Qin J, Li R, Raes J, et al. A human gut microbial gene catalogue established by metagenomic sequencing. Nature 2010;464: 59-65

5. Human Microbiome Project Consortium. A framework for human microbiome research. Nature 2012;486:215-21
6. Peterson J, Garges S, Giovanni M, et al. The NIH Human Microbiome Project. Genome Res 2009;19:2317-23

7. Dethlefsen L, Eckburg PB, Bik EM, Relman DA. Assembly of the human intestinal microbiota. Trends Ecol Evol 2006;21:517-23

8. Turnbaugh PJ, Ley RE, Hamady M, et al. The human microbiome project. Nature 2007;449:804-10

9. Guarner F, Malagelada JR. Gut flora in health and disease. Lancet 2003;361:512-19

10. Turnbaugh PJ, Ley RE, Mahowald MA, et al. An obesity-associated gut microbiome with increased capacity for energy harvest. Nature 2006;444:1027-31

11. Yang L, Lu X, Nossa CW, et al. Inflammation and intestinal metaplasia of the distal esophagus are associated with alterations in the microbiome. Gastroenterology 2009;137:588-97
12. Vijay-Kumar M, Aitken JD, Carvalho FA, et al. Metabolic syndrome and altered gut microbiota in mice lacking Toll-like receptor 5. Science 2010;328:228-31

13. Maldonado-Contreras A, Goldfarb KC, Godoy-Vitorino F, et al. Structure of the human gastric bacterial community in relation to Helicobacter pylori status. Isme J 2011;5:574-9

14. Marchesi JR, Dutilh BE, Hall N, et al. Towards the human colorectal cancer microbiome. PLoS One 2011;6:e20447

15. Fukuda $S$, Toh $\mathrm{H}$, Hase K, et al. Bifidobacteria can protect from enteropathogenic infection through production of acetate. Nature 2011;469: 543-7

16. Arthur JC, Perez-Chanona E, Muhlbauer M, et al. Intestinal inflammation targets cancer-inducing activity of the microbiota. Science 2012;338:120-3

17. Corr SC, Li Y, Riedel CU, et al. Bacteriocin production as a mechanism for 
the antiinfective activity of Lactobacillus salivarius UCC118. Proc Natl Acad Sci USA 2007;104:7617-21

18. Ghosh S, Dai C, Brown K, et al. Colonic microbiota alters host susceptibility to infectious colitis by modulating inflammation, redox status, and ion transporter gene expression. Am J Physiol Gastrointest Liver Physiol 2011;301:G39-49

19. Tomasello G, Bellavia M, Palumbo VD, et al. From gut microflora imbalance to mycobacteria infection: is there a relationship with chronic intestinal inflammatory diseases? Ann Ital Chir 2011;82:361-8

20. Sartor RB. Microbial influences in inflammatory bowel diseases. Gastroenterology 2008;134:577-94

21. Elson CO, Cong Y, McCracken VJ, et al. Experimental models of inflammatory bowel disease reveal innate, adaptive, and regulatory mechanisms of host dialogue with the microbiota. Immunol Rev 2005;206: 260-76

22. Khan AA, Shrivastava A. Bacterial infections associated with cancer: possible implication in etiology with special reference to lateral gene transfer. Cancer Metastasis Rev 2010;29:331-7

23. Michaud DS, Izard J, Wilhelm-Benartzi CS, et al. Plasma antibodies to oral bacteria and risk of pancreatic cancer in a large European prospective cohort study. Gut 2013;62: 1764-70

24. Farrell JJ, Zhang L, Zhou H, et al. Variations of oral microbiota are associated with pancreatic diseases including pancreatic cancer. Gut 2012;61:582-8

25. Pushalkar S, Ji X, Li Y, et al. Comparison of oral microbiota in tumor and non-tumor tissues of patients with oral squamous cell carcinoma. BMC Microbiol 2012;12:144

26. Mager DL, Haffajee AD, Devlin PM, et al. The salivary microbiota as a diagnostic indicator of oral cancer: a descriptive, non-randomized study of cancer-free and oral squamous cell carcinoma subjects. J Transl Med 2005;3:27

27. Wu S, Rhee KJ, Albesiano E, et al. A human colonic commensal promotes colon tumorigenesis via activation of $\mathrm{T}$ helper type $17 \mathrm{~T}$ cell responses. Nat Med 2009;15:1016-22

28. Tjalsma H, Boleij A, Marchesi JR, Dutilh BE. A bacterial driver-passenger model for colorectal cancer: beyond the usual suspects. Nat Rev Microbiol 2012;10: 575-82
29. Zhu Q, Jin $\mathrm{Z}, \mathrm{Wu}$ W, et al. Analysis of the intestinal lumen microbiota in an animal model of colorectal cancer. PLoS One 2014;9:e90849

30. Kostic AD, Gevers D, Pedamallu CS, et al Genomic analysis identifies association of Fusobacterium with colorectal carcinoma. Genome Res 2012;22:292-8

31. Geng J, Fan H, Tang X, et al. Diversified pattern of the human colorectal cancer microbiome. Gut Pathog 2013;5:2

32. Castellarin M, Warren RL, Freeman JD, et al. Fusobacterium nucleatum infection is prevalent in human colorectal carcinoma. Genome Res 2012;22:299-306

33. Ahn J, Sinha R, Pei Z, et al. Human gut microbiome and risk for colorectal cancer. J Natl Cancer Inst 2013;105:1907-11

34. Fukase K, Kato M, Kikuchi S, et al. Effect of eradication of Helicobacter pylori on incidence of metachronous gastric carcinoma after endoscopic resection of early gastric cancer: an open-label, randomised controlled trial. Lancet 2008;372:392-7

35. Giannakis M, Backhed HK, Chen SL, et al. Response of gastric epithelial progenitors to Helicobacter pylori Isolates obtained from Swedish patients with chronic atrophic gastritis. J Biol Chem 2009;284:30383-94

36. Compare D, Nardone G. Contribution of gut microbiota to colonic and extracolonic cancer development. Dig Dis 2011;29: 554-61

37. Mishra RR, Tewari M, Shukla HS. Helicobacter pylori and pathogenesis of gallbladder cancer. J Gastroenterol Hepatol 2011;26:260-6

38. Wang L, Zollinger T, Zhang J. Association between Helicobacter pylori infection and liver cancer mortality in 67 rural Chinese counties. Cancer Causes Control 2013;24: 1331-7

39. Swisher SC, Barbati AJ. Helicobacter pylori strikes again: gastric mucosa-associated lymphoid tissue (MALT) lymphoma. Gastroenterol Nurs 2007;30:348-54; quiz 355-346

40. Nie S, Chen T, Yang X, et al. Association of Helicobacter pylori infection with esophageal adenocarcinoma and squamous cell carcinoma: a meta-analysis. Dis Esophagus 2014

41. Narikiyo M, Tanabe C, Yamada Y, et al. Frequent and preferential infection of Treponema denticola, Streptococcus mitis, and Streptococcus anginosus in esophageal cancers. Cancer Sci 2004;95:569-74
42. Kim NH, Park JP, Jeon SH, et al. Purulent pericarditis caused by group $\mathrm{G}$ streptococcus as an initial presentation of colon cancer. J Korean Med Sci 2002;17:571-3

43. Macfarlane S, Furrie E, Macfarlane GT, Dillon JF. Microbial colonization of the upper gastrointestinal tract in patients with Barrett's esophagus. Clin Infect Dis 2007; 45:29-38

44. Balkwill F, Mantovani A. Inflammation and cancer: back to Virchow? Lancet 2001;357: 539-45

45. Iida N, Dzutsev A, Stewart CA, et al. Commensal bacteria control cancer response to therapy by modulating the tumor microenvironment. Science 2013;342: 967-70

46. Greenhill C. Gut microbiota: anti-cancer therapies affected by gut microbiota. Nat Rev Gastroenterol Hepatol 2014;11:1

47. Grote VA, Kaaks R, Nieters A, et al. Inflammation marker and risk of pancreatic cancer: a nested case-control study within the EPIC cohort. Br J Cancer 2012;106: 1866-74

48. Wang L, Jiang Y, Zhang Y, et al. Association analysis of IL-17A and IL-17F polymorphisms in Chinese Han women with breast cancer. PLoS One 2012;7: e34400

49. de Martel C, Ferlay J, Franceschi S, et al Global burden of cancers attributable to infections in 2008: a review and synthetic analysis. Lancet Oncol 2012;13:607-15

50. Xie FJ, Zhang YP, Zheng QQ, et al. Helicobacter pylori infection and esophageal cancer risk: an updated meta-analysis. World J Gastroenterol 2013;19:6098-107

51. Nagamine CM, Rogers AB, Fox JG, Schauer DB. Helicobacter hepaticus promotes azoxymethane-initiated colon tumorigenesis in BALB/c-IL10-deficient mice. Int J Cancer 2008;122:832-8

52. Marteau P, Chaput U. Bacteria as trigger for chronic gastrointestinal disorders. Dig Dis 2011;29:166-71

53. Khan MA, Jakate $S$, Komanduri S. Rare AIDS-associated plasmablastic lymphoma as the initial presentation of AIDS. Clin Adv Hematol Oncol 2010;8:55-7

54. Ozdemir H, Ciftci E, Karbuz A, et al. Successful treatment of multidrug-resistant Escherichia coli bacteremia with tigecycline in an acute myeloid leukemia child. Turk J Pediatr 2012;54:59-60

55. Huynh ML, Fadok VA, Henson PM. Phosphatidylserine-dependent ingestion of apoptotic cells promotes 
TGF-beta1 secretion and the resolution of inflammation. J Clin Invest 2002;109:41-50

56. Gabillet J, Millet A, Pederzoli-Ribeil M, et al. Proteinase 3, the autoantigen in granulomatosis with polyangiitis, associates with calreticulin on apoptotic neutrophils, impairs macrophage phagocytosis, and promotes inflammation. J Immunol 2012;189:2574-83

57. Protti MP, De Monte L. Cross-talk within the tumor microenvironment mediates Th2-type inflammation in pancreatic cancer. Oncoimmunology 2012;1:89-91

58. Li S, Wang N, Brodt P. Metastatic cells can escape the proapoptotic effects of TNF-alpha through increased autocrine IL6/STAT3 signaling. Cancer Res 2012;72: 865-75

59. Sorensen LT. Wound healing and infection in surgery: the pathophysiological impact of smoking, smoking cessation, and nicotine replacement therapy: a systematic review. Ann Surg 2012;255:1069-79

60. Maiuri MC, Tajana G, Iuvone T, et al. Nuclear factor-kappa B regulates inflammatory cell apoptosis and phagocytosis in rat carrageenan-sponge implant model. Am J Pathol 2004;165: $115-26$

61. Ward SV, Cadby G, Heyworth JS, et al. Association of TGFbeta1 and clinical factors with scar outcome following melanoma excision. Arch Dermatol Res 2012;304: 343-51

62. Joo CK, Seomun Y. Matrix metalloproteinase (MMP) and TGF beta 1 -stimulated cell migration in skin and cornea wound healing. Cell Adh Migr 2008;2:252-3

63. Ganesh K, Das A, Dickerson R, et al. Prostaglandin E(2) induces oncostatin $M$ expression in human chronic wound macrophages through Axl receptor tyrosine kinase pathway. J Immunol 2012;189: 2563-73

64. Lakshmi RT, Priyanka T, Meenakshi J, et al. Low molecular weight heparin mediated regulation of nitric oxide synthase during burn wound healing. Ann Burns Fire Disasters 2011;24:24-9

65. Bitar MS, Al-Mulla F. ROS constitute a convergence nexus in the development of IGF1 resistance and impaired wound healing in a rat model of type 2 diabetes. Dis Model Mech 2012;5:375-88

66. Duckworth CA, Clyde D, Pritchard DM. CD24 is expressed in gastric parietal cells and regulates apoptosis and the response to Helicobacter felis infection in the murine stomach. Am J Physiol Gastrointest Liver Physiol 2012;303:G915-26

67. Pandey M, Gupta KP. Involvement of STAT3, NF-kappa B and associated downstream molecules before and after the onset of urethane induced lung tumors in mouse. Environ Toxicol Pharmacol 2012;34:502-11

68. Saito K, Kihara K. Role of C-reactive protein in urological cancers: a useful biomarker for predicting outcomes. Int J Urol 2013;20:161-71

69. Philip M, Rowley DA, Schreiber H. Inflammation as a tumor promoter in cancer induction. Semin Cancer Biol 2004;14:433-9

70. Macarthur M, Hold GL, El-Omar EM. Inflammation and Cancer II. Role of chronic inflammation and cytokine gene polymorphisms in the pathogenesis of gastrointestinal malignancy. Am J Physiol Gastrointest Liver Physiol 2004;286: G515-20

71. Tretyakova NY, Burney S, Pamir B, et al. Peroxynitrite-induced DNA damage in the supF gene: correlation with the mutational spectrum. Mutat Res 2000;447:287-303

72. Mutamba JT, Svilar D, Prasongtanakij S, et al. XRCC1 and base excision repair balance in response to nitric oxide. DNA Repair (Amst) 2011;10:1282-93

73. Shimizu T, Marusawa H, Endo Y, Chiba T. Inflammation-mediated genomic instability: roles of activation-induced cytidine deaminase in carcinogenesis. Cancer Sci 2012;103:1201-6

74. Pollard JW. Tumour-educated macrophages promote tumour progression and metastasis. Nat Rev Cancer 2004;4:71-8

75. Petrenko O, Moll UM. Macrophage migration inhibitory factor MIF interferes with the Rb-E2F pathway. Mol Cell 2005; 17:225-36

76. Gesser B, Rasmussen MK, Raaby L, et al. Dimethylfumarate inhibits MIF-induced proliferation of keratinocytes by inhibiting MSK1 and RSK1 activation and by inducing nuclear p-c-Jun (S63) and p-p53 (S15) expression. Inflamm Res 2011;60: 643-53

77. McCarron AJ, Armstrong C, Glynn G, et al. Antibacterial effects on acinetobacter species of commonly employed antineoplastic agents used in the treatment of haematological malignancies: an in vitro laboratory evaluation. Br J Biomed Sci 2012;69:14-17

78. Prabhash K, Medhekar A, Ghadyalpatil N, et al. Blood stream infections in cancer patients: a single center experience of isolates and sensitivity pattern. Indian J Cancer 2010;47:184-8

79. Chiang MC, Kuo SC, Chen SJ, et al. Clinical characteristics and outcomes of bacteremia due to different genomic species of Acinetobacter baumannii complex in patients with solid tumors. Infection 2012;40:19-26

80. Roperto S, Di Guardo G, Leonardi L, et al. Bacterial isolates from the urine of cattle affected by urothelial tumors of the urinary bladder. Res Vet Sci 2012;93:1361-6

81. Yang L, Francois F, Pei Z. Molecular pathways: pathogenesis and clinical implications of microbiome alteration in esophagitis and Barrett esophagus. Clin Cancer Res 2012;18:2138-44

82. Fan YP, Chakder S, Gao F, Rattan S. Inducible and neuronal nitric oxide synthase involvement in lipopolysaccharide-induced sphincteric dysfunction. Am J Physiol Gastrointest Liver Physiol 2001;280:G32-42

83. Calatayud S, Garcia-Zaragoza E, Hernandez C, et al. Downregulation of nNOS and synthesis of PGs associated with endotoxin-induced delay in gastric emptying. Am J Physiol Gastrointest Liver Physiol 2002;283:G1360-7

84. Smolinska MJ, Page TH, Urbaniak AM, et al. Hck tyrosine kinase regulates TLR4-induced TNF and IL- 6 production via AP-1. J Immunol 2011;187:6043-51

85. Jarchum I, Liu M, Shi C, et al. Critical role for MyD88-mediated neutrophil recruitment during Clostridium difficile colitis. Infect Immun 2012;80:2989-96

86. Chinen T, Volchkov PY, Chervonsky AV, Rudensky AY. A critical role for regulatory $\mathrm{T}$ cell-mediated control of inflammation in the absence of commensal microbiota. J Exp Med 2010;207:2323-30

87. Carvalho FA, Aitken JD, Vijay-Kumar M, Gewirtz AT. Toll-like receptor-gut microbiota interactions: perturb at your own risk!. Annu Rev Physiol 2010;74:177-98

88. Kinross J, von Roon AC, Penney N, et al. The gut microbiota as a target for improved surgical outcome and improved patient care. Curr Pharm Des 2009;15:1537-45

89. Ubeda C, Taur Y, Jenq RR, et al. Vancomycin-resistant Enterococcus domination of intestinal microbiota is enabled by antibiotic treatment in mice and precedes bloodstream invasion in humans. J Clin Invest 2010;120:4332-41

90. Merline R, Moreth K, Beckmann J, et al. Signaling by the matrix proteoglycan decorin controls inflammation and cancer 
through PDCD4 and MicroRNA-21. Sci Signal 2011;4:ra75

91. Moreth K, Iozzo RV, Schaefer L. Small leucine-rich proteoglycans orchestrate receptor crosstalk during inflammation. Cell Cycle 2012;11:2084-91

92. Gao Q, Zhao YJ, Wang XY, et al. CXCR6 upregulation contributes to a proinflammatory tumor microenvironment that drives metastasis and poor patient outcomes in hepatocellular carcinoma. Cancer Res 2012;72:3546-56

93. Uronis JM, Muhlbauer M, Herfarth HH, et al. Modulation of the intestinal microbiota alters colitis-associated colorectal cancer susceptibility. PLoS One 2009; 4: e6026

94. Rogers AB, Fox JG. Inflammation and Cancer. I. Rodent models of infectious gastrointestinal and liver cancer. Am J Physiol Gastrointest Liver Physiol 2004;286: G361-6

95. Meurman JH. Oral microbiota and cancer. J Oral Microbiol 2010;2

96. Pizzo G, Guiglia R, Lo Russo L, Campisi G. Dentistry and internal medicine: from the focal infection theory to the periodontal medicine concept. Eur J Intern Med 2010;21:496-502

97. Round JL, Lee SM, Li J, et al. The Toll-like receptor 2 pathway establishes colonization by a commensal of the human microbiota. Science 2011;332:974-7

98. Sheyhidin I, Nabi G, Hasim A, et al. Overexpression of TLR3, TLR4, TLR7 and TLR9 in esophageal squamous cell carcinoma. World J Gastroenterol 2011;17: 3745-51

99. Zhang YB, He FL, Fang M, et al. Increased expression of Toll-like receptors 4 and 9 in human lung cancer. Mol Biol Rep 2009;36: 1475-81

100. Rakoff-Nahoum S, Medzhitov R. Toll-like receptors and cancer. Nat Rev Cancer 2009;9:57-63

101. Fukata M, Abreu MT. Role of Toll-like receptors in gastrointestinal malignancies. Oncogene 2008;27:234-43

102. Tlaskalova-Hogenova H, Stepankova R, Kozakova $\mathrm{H}$, et al. The role of gut microbiota (commensal bacteria) and the mucosal barrier in the pathogenesis of inflammatory and autoimmune diseases and cancer: contribution of germ-free and gnotobiotic animal models of human diseases. Cell Mol Immunol 2011;8:110-20

103. Bauer E, Williams BA, Smidt H, et al. Influence of the gastrointestinal microbiota on development of the immune system in young animals. Curr Issues Intest Microbiol 2006;7:35-51

104. Ma EL, Choi YJ, Choi J, et al. The anticancer effect of probiotic Bacillus polyfermenticus on human colon cancer cells is mediated through ErbB2 and ErbB3 inhibition. Int J Cancer 2010;127: 780-90

105. Jia W, Li H, Zhao L, Nicholson JK. Gut microbiota: a potential new territory for drug targeting. Nat Rev Drug Discov 2008;7:123-9

106. Naglova H, Bucova M. HMGB1 and its physiological and pathological roles. Bratisl Lek Listy 2012;113:163-71

107. Wang YZ, Yan M, Tian FF, et al. Possible involvement of toll-like receptors in the pathogenesis of myasthenia gravis. Inflammation 2012;36:121-30

108. Belinda LW, Wei WX, Hanh BT, et al. SARM: a novel Toll-like receptor adaptor, is functionally conserved from arthropod to human. Mol Immunol 2008;45:1732-42

109. Valkov E, Stamp A, Dimaio F, et al. Crystal structure of Toll-like receptor adaptor MAL/TIRAP reveals the molecular basis for signal transduction and disease protection. Proc Natl Acad Sci USA 2011;108:14879-84

110. Kawai T, Akira S. TLR signaling. Semin Immunol 2007;19:24-32

111. Moossavi S, Rezaei N. Toll-like receptor signalling and their therapeutic targeting in colorectal cancer. Int Immunopharmacol 2013;16:199-209

112. Hedayat M, Takeda K, Rezaei N. Prophylactic and therapeutic implications of toll-like receptor ligands. Med Res Rev 2012;32:294-325

113. Hofmann MA, Kors C, Audring H, et al. Phase 1 evaluation of intralesionally injected TLR9-agonist PF-3512676 in patients with basal cell carcinoma or metastatic melanoma. J Immunother 2008;31:520-7

114. Pashenkov M, Goess G, Wagner C, et al. Phase II trial of a toll-like receptor 9-activating oligonucleotide in patients with metastatic melanoma. J Clin Oncol 2006;24:5716-24

115. Thompson JA, Kuzel T, Drucker BJ, et al. Safety and efficacy of PF-3512676 for the treatment of stage IV renal cell carcinoma: an open-label, multicenter phase I/II study. Clin Genitourin Cancer 2009;7:E58-65

116. Kim YH, Girardi M, Duvic M, et al Phase I trial of a Toll-like receptor 9 agonist, PF-3512676 (CPG 7909), in patients with treatment-refractory, cutaneous T-cell lymphoma. J Am Acad Dermatol 2010;63:975-83

117. Burns CA, Brown MD. Imiquimod for the treatment of skin cancer. Dermatol Clin 2005;23:151-64; vii

118. Witt PL, Ritch PS, Reding D, et al. Phase I trial of an oral immunomodulator and interferon inducer in cancer patients. Cancer Res 1993;53:5176-80

119. Khan AA, Shrivastava A, Khurshid M. Normal to cancer microbiome transformation and its implication in cancer diagnosis. Biochim Biophys Acta 2012;1826:331-7

120. Eckburg PB, Bik EM, Bernstein CN, et al. Diversity of the human intestinal microbial flora. Science 2005;308:1635-8

121. Lampe JW. The Human Microbiome Project: getting to the guts of the matter in cancer epidemiology. Cancer Epidemiol Biomarkers Prev 2008;17:2523-4

122. Tannock GW. Analysis of the intestinal microflora using molecular methods. Eur J Clin Nutr 2002;4(56 Suppl):S44-9

123. Pagnini C, Corleto VD, Mangoni ML, et al. Alteration of local microflora and alpha-defensins hyper-production in colonic adenoma mucosa. J Clin Gastroenterol 2011;45:602-10

124. Kulasingam V, Diamandis EP. Strategies for discovering novel cancer biomarkers through utilization of emerging technologies. Nat Clin Pract Oncol 2008;5:588-99

125. Hord NG. Eukaryotic-microbiota crosstalk: potential mechanisms for health benefits of prebiotics and probiotics. Annu Rev Nutr 2008;28:215-31

126. Hayes DF, Bast RC, Desch CE, et al. Tumor marker utility grading system: a framework to evaluate clinical utility of tumor markers. J Natl Cancer Inst 1996;88: 1456-66

127. Sturgeon CM, Hoffman BR, Chan DW, et al. National Academy of Clinical Biochemistry Laboratory Medicine Practice Guidelines for use of tumor markers in clinical practice: quality requirements. Clin Chem 2008;54:e1-e10

128. Koomen JM, Li D, Xiao LC, et al. Direct tandem mass spectrometry reveals limitations in protein profiling experiments for plasma biomarker discovery. J Proteome Res 2005;4:972-81

129. Amatschek S, Koenig U, Auer H, et al. Tissue-wide expression profiling using cDNA subtraction and microarrays to 


\section{Review Shahanavaj, Gil Bazo, Castiglia et al.}

identify tumor-specific genes. Cancer Res 2004;64:844-56

130. Michiels S, Koscielny S, Hill C. Prediction of cancer outcome with microarrays: a multiple random validation strategy. Lancet 2005;365:488-92

131. Hirayama K, Rafter J. The role of probiotic bacteria in cancer prevention. Microbes Infect 2000;2:681-6

132. Schmitt M, Depuydt C, Benoy I, et al. Viral load of high-risk human papillomaviruses as reliable clinical predictor for the presence of cervical lesions. Cancer Epidemiol Biomarkers Prev 2013;22:406-14

133. Schmitt M, Depuydt C, Benoy I, et al. Prevalence and viral load of 51 genital human papillomavirus types and three subtypes. Int J Cancer 2013;132(10): 2395-403

134. Diamandis EP. Present and future of cancer biomarkers. Clin Chem Lab Med 2014;52: $791-4$

135. Lee J.E, Lee S, Lee H, et al. Association of the vaginal microbiota with human papillomavirus infection in a Korean twin cohort. PLoS One 2013;8:e63514

136. Grivennikov S.I, Wang K, Mucida D, et al. Adenoma-linked barrier defects and microbial products drive IL-23/IL-17mediated tumour growth. Nature 2012;491: 254-8

137. Swidsinski A, Khilkin M, Kerjaschki D, et al. Association between intraepithelial Escherichia coli and colorectal cancer. Gastroenterology 1998;115:281-6

138. Shen X.J, Rawls J.F, Randall T, et al. Molecular characterization of mucosal adherent bacteria and associations with colorectal adenomas. Gut Microbes 2010;1: 138-47

139. Sobhani I, Tap J, Roudot-Thoraval F, et al. Microbial dysbiosis in colorectal cancer (CRC) patients. PLoS One 2011;6:e16393

140. Sasaki M, Yamaura C, Ohara-Nemoto Y, et al. Streptococcus anginosus infection in oral cancer and its infection route. Oral Dis 2005;11:151-6
141. Sasaki H, Ishizuka T, Muto M, et al. Presence of Streptococcus anginosus DNA in esophageal cancer, dysplasia of esophagus, and gastric cancer. Cancer Res 1998;58: 2991-5

142. Anderson L.A, Murphy S.J, Johnston B.T, et al. Relationship between Helicobacter pylori infection and gastric atrophy and the stages of the oesophageal inflammation, metaplasia, adenocarcinoma sequence: results from the FINBAR case-control study. Gut 2008;57:734-9

143. Muller L.B, Fagundes R.B, Moraes C.C. Rampazzo A. [Prevalence of Helicobacter pylori infection and gastric cancer precursor lesions in patients with dyspepsia]. Arq Gastroenterol 2007;44:93-8

144. Sharma V, Chauhan V.S, Nath G, et al. Role of bile bacteria in gallbladder carcinoma. Hepatogastroenterology 2007;54: $1622-5$ 